\title{
An Approach Towards Dynamic Opportunistic Routing in Wireless Mesh Networks
}

\author{
Sudhanshu Kulshrestha, Aditya Trivedi \\ Department of Information and Communication Technology, ABV-Indian Institute of Information Technology \\ and Management, Gwalior, India-474010
}

\begin{abstract}
Opportunistic routing (OR) for multi-hop wireless networks was first proposed by Biswas and Morris in 2004, but again as a modified version in 2005 as Extremely Opportunistic Routing (ExOR). A few other variants of the same were also proposed in the meanwhile time. In this paper we propose a Dynamic Opportunistic Routing (DOR) protocol which depends on network density and also provides spatial diversity. Our routing protocol is distributed in nature and provides partial 802.11 MAC layer abstraction. To verify the results of our protocol we took a network with light-density of nodes and bigger in size (as OR performs better in higher node density). A wireless mesh network in "QualNet network simulator" was created, where the average endto-end delay and throughput at every node are compared with that of other standard routing protocol OLSRINRIA.
\end{abstract}

Index Terms: Extremely opportunistic routing; IEEE 802.11 MAC; wireless routing; bellmanford algorithm; radio transmission range

(C) 2012 Published by MECS Publisher. Selection and/or peer review under responsibility of the Research Association of Modern Education and Computer Science

\section{Introduction}

This paper presents a Dynamic Opportunistic Routing (DOR), which is actually based on the Extremely Opportunistic Routing (ExOR) [1], but somewhat functionally different. Opportunistic routing (OR) [2], [3], [4], [5], [6], [7] is among one of the best routing solution to multi-hop wireless ad-hoc networks. Most of the previous version of ORs had a common thing in general, that they needed to schedule and synchronize all the candidate opportunistic forwarders, to decide for the actual forwarder [1], [5], [6]. Although many different ORs have been proposed so far, but still there's a need of a versatile routing which would outperform the predefined routing protocol in practical distortion phenomenon such as fading, shadowing, etc during simulation and empirical measurements. Under the motive of designing such a protocol, we have introduced Dynamic Opportunistic Routing which would comprise following:

* Corresponding author.

E-mail address: atrivedi@iiitm.ac.in, sudhanshu.kulshrestha@students.iiitm.ac.in 
- Dynamicity in the sense that path selection should be dependent on transmission distance and node density.

- Spatial diversity (as it would not alter the 802.11 MAC standards, so that it could be deployed in alternate traffic types e.g. multicast).

- Minimal size of candidate forwarder-set (to ensure that the overall overhead of this distributed algorithm is kept low).

The rest of this paper is organized as follows: the Section II provides the motivation of designing this routing protocol. Section III reviews the related work done so far, with emphasis on their functionality. Section IV describes the Dynamic Opportunistic Routing protocol in detail. Section V is devoted to implementation details and the results. Last Section V allows us to conclude this paper and gives the direction of future work.

\section{Motivation}

\section{A. Opportunism of Exploring Potential Forwarding Nodes}

In opportunistic routing, broadcast nature of wireless transmission is exploited by making decisions in an online manner, choosing the next relay based on actual transmission outcomes and as well as a rank ordering of relays. Although the optimal routing decision at any epoch is to select the next relay node based on an index summarizing the expected cost-to-forward from current node to the destination. However this cost should include various parameters such as current queue backlogs, distortion mechanisms, congestion information, shortest path calculation etc.

\section{B. Performance of Opportunistic Routing}

The performance of opportunistic routing is sensitive to three major points [1], [5], [6]: (i) Node densityhigher is better. (ii) Length of the path- longer is better. (iii) Rate at which packet reception probability falls off with distance. But it is noticeable that, as the size of the network and also the density increases, the candidate set size would also increase, refer Fig 1. This in turn makes the co-ordination mechanism, slotted 802.11 MAC [1] or random packet coding [6] or any other, to create a higher overhead to synchronize them for deciding upon the actual forwarder [1] or the innovative packet [6]. Thus, there arises a need to restrict the complexity up to a particular threshold and also to make the routing protocol dynamic so that it could switch between tradition best path routing (if it is a low density and short distance transmission) and opportunistic routing.

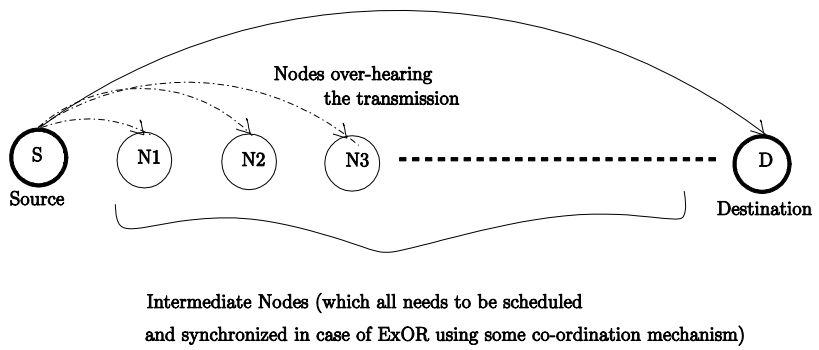

Figure 1. Typical Opportunistic Routing, where in many cases a large no. of intermediate nodes would have to be synchronized.

\section{Related Work}

In recent years, there has been a keen interest for designing opportunistic routing protocol for multi-hop wireless ad-hoc networks, which could perform best in majority of the scenarios. In ExOR [1] in 2005, which outperformed the standard predefined routing (Dijkstra's shortest path algorithm) by almost more than 50\%, the packets are broadcast to a candidate set based on the shortest number of hops and the high priority node 
(prioritized by delivery rates) is expected to forward the same towards the destination. As, ExOR compromised the 802.11 MAC and thus spatial diversity, each forwarder had to wait for a total no as many number of time slots as there are forwarding candidates in the received packet. This requires a delivery ratio matrix containing an approximation of the loss rate for direct radio transmission between every pair of nodes, to be maintained at each node. This matrix is built using a link-state flooding scheme, in which nodes measure loss rates and periodically flood statistics updates. Also, slotted 802.11 MAC is used to reserve multiple slots of time as per priority of nodes, which in turn increased the overhead of the nodes by $8.3 \%$ as compared to unicast 802.11 , due to frame modification.

In 2007 authors of [6] introduced MAC independent Opportunistic Routing and Encoding (MORE). It does not need any special scheduler to coordinate routers and thus exploiting spatial reuse, thereby running on top of 802.11. Encoding refers to "Random Linear Combination" of packets done in polynomial time. Here, the file is broken into batches, which further into ' $\mathrm{K}$ ' packets ( $\mathrm{K}$ may vary for each batch). Coding is done at source and then broadcast after attaching a variable length MORE header. Only "Code Vector" field in MORE header is computed locally at each forwarder, rest fields are copied as arrived. At each next hop, if it is there in the packet's forwarder list; linear independence of packets is checked (Gaussian elimination). Only innovative packets are accepted for current batch, otherwise discarded. And if the batch no. is changed, then all the packets are accepted after flushing the batch buffer (Batch buffer is also flushed and updated in case if nodes overhear an $\mathrm{ACK}$ ). Destination decodes the whole batch (matrix inversion) after receiving $\mathrm{K}$ innovative packets and sends back ACK.

In 2008, authors of [8], took an analytical approach to study the potential gain of opportunistic routing in multi hop wireless networks. They followed the non-deterministic model of channel under the diverse radio propagation environment using lognormal shadowing and Rayleigh fading models.

In 2009, authors of [5] introduced Local Scheduling Scheme for Opportunistic Routing; they considered the whole network as a graph and partitioned it into sub-graphs (hops). Thus left with lesser no of hops to compute the forwarding set. Within the sub-graphs, ExOR is used. And for equivalent set (sub-graphs as hops), a metric based method (ETX [9]) is employed to compute the fixed predetermined path. Graph is partitioned such that a pair of nodes with in adjacent sub-graphs should be highly uncorrelated. And then they minimized the no. of such edges (cut). Finally an algorithm is proposed so that multiple transmissions can be done at an instant i.e. same single packet, in different radio range, can traverse the network at the same time. Simulation is done under six set of topologies, variable size, to compare with ExOR.

In 2009, authors of [7] proposed a mathematical model. Adaptive Opportunistic Routing does not rely upon channel statistics and network model. For reference, author has chosen probabilistic local broadcast model, where the optimum routing policies depends on transmission success probabilities. Obeying the Markov Decision Theoretic approach, a reward system is maintained. After that, the average per packet reward associated with ' $m$ ' packets within ' $n$ ' time slots, for a particular set of nodes $S \in \Phi$ is computed. Then this average (mean) value is proved to be converging to the solution of Auxiliary Problem and is optimal also.

Naghshvar and Javidi [4] introduced recently in 2010, multiple sources of traffic and wireless links with stochastic reliability based Opportunistic Routing with Congestion Diversity (ORCDs) viz. P-ORCD, InfreqORCD and D-ORCD. Scheduling (a three step transmission process) of mostly all opportunistic algorithms depends upon the cost measure assigned to each node. But a trade-off is always there between "opportunism" (diversity gain) and "control and computation overhead". Their brief details are:

- P-ORCD: An upper limit to the size of forwarding set (will be called as best neighbors) is imposed, thereby decreasing overhead and thus diversity gain.

- Infreq-ORCD: Centralized congestion cost measures are computed periodically with a time period less than length of routing decision cycle of ORCD, thereby decreasing computation cost at every node in each time slot. Algorithm as same as Dijkstra's is employed.

- D-ORCD: Decentralized cost measurement is done iteratively at each node where the information of the next possible hop only is considered, thereby restricting the 'space'. 


\section{Details of Protocol}

Our proposed routing protocol uses a very simple functionality. It is a distributed and recursive protocol, i.e. it calls for itself at each forwarder. It first considers a maximum radio transmission range for every node in the network, out of

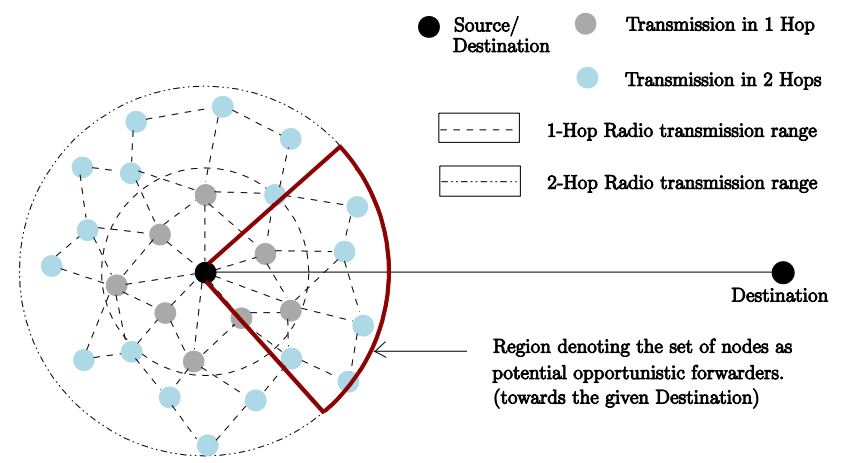

Figure 2. New proposed functionality, with restricted but definite opportunism.

which the probability of successful direct packet reception becomes very small. We named it as 1-Hop radio transmission range. Then we again find out the 1-Hop radio transmission range of those nodes whose are actually itself in the 1-Hop transmission range of the source node i. We named this as 2-Hop radio transmission range, which comprises of all those nodes to whom source node i can transmit the packets in 'at least' 2 hops, refer to Fig 2.

\section{Data Structures and Proposed Algorithm}

This is a kind of proactive routing protocol, where an undirected link (i,j) connecting two nodes $\mathrm{i}$ and $\mathrm{j}$ is formed when the distance becomes less than or equal to the 1-Hop radio transmission range. For every node i, one list and two tables are formed. The List 1 contains all the neighboring 1-Hop nodes. Whereas the Table 1 is to store the current topology, which is based on the periodic link-state information received from neighboring nodes. Table 2 is to store the 1-Hop nodes for every particular destination $\mathrm{j}$ via mapping to the List 1 ; along with the 2-Hop nodes for the current source node $i$ that too via mapping to the List 1 for 1-Hop nodes of $i$ those are already in the Table 2. This is a redundant procedure and finally the Table 2 has the listing of the neighboring nodes in an order according to distance for every possible destination $\mathrm{j}$. The DOR protocol works as follows:

1) Identify all the neighbors with in the 1-Hop and 2-Hop radio transmission range respectively.

2) Then the next step is to identify all those nodes which are towards the direction of the destination. This is done using the mapping with in user-defined data-structures and the standard data-structures, e.g. those used in storing the distance of shortest path from source to destination. Also, if this count of nodes is below a particular threshold value $\eta$, then redirect the control to the standard predefined best path routing (Bellmanford algorithm). This is done because it is reported that opportunistic routing does not performs so well under low density of nodes [1] [5] [8].

3) This is a crucial step which accounts for the lower complexity of the algorithm. Here, just exactly 3 candidates are chosen from with in the set of potential opportunistic forwarders. The three candidates are chosen according to following rules (to be chosen from the tables Table 1 and Table 2 formed in step (2)):

- Candidate 1: Farthest from the source, but with-in 1-Hop radio transmission range.

- Candidate 2: Farthest from the source other than Candidate 1, but with-in 1-Hop radio transmission range.

- Candidate 3: Closest to the source, but with-in 2-Hop radio transmission range. 
4) The candidates chosen in step (3) are then added to the data frame format. But we did not compromise the spatial diversity as all of these forwarders can forward concurrently. And most of the times, the nodes in next candidate set chosen by current 3-candidates are same; this helps to reduce the redundant traffic. Now the packet is transmitted to the destination and a variable timer is started according to the iteration of these steps. The timer is a linear variable and is inversely proportional to the number of the iteration of these steps (as this is a distributed and recursive protocol). We see that as the packet will progress towards the destination, the timer value will decrease. And after the time out, the packets will be resent from that epoch.

5) These steps (1) to (4) continue to be repeated until the ultimate destination is reached by the packet.

\section{Implementation Details and Results}

\section{Simulation Model}

The simulation study was carried out using QualNet 5.0 network simulator [10]. We created the scenarios as a wireless mesh network [11]. We took 50 nodes placed randomly in a $1000 \mathrm{~m} \times 1000 \mathrm{~m}$ grid. Our intention was to test the protocol under less denser network. Out of those 50 nodes, terminal nodes were acting like mesh clients, while others as mesh points. We considered only the IPv4 network protocol. Constant Bit Rate (CBR) traffic was generated at a node on the edge of the network with a number of destinations; some being near-by nodes, while some were at opposite side of the grid. Intention was that the amount of traffic faced at most of the points in the network should be reasonably constant. In addition, circular radio range model was assumed. Other simulation parameter details are given in Table 1 as follows:

TABLE I. SIMULATION PARAMETERS

\begin{tabular}{|l|l|}
\hline \multicolumn{1}{|c|}{ Parameter } & \multicolumn{1}{c|}{ Value } \\
\hline Path loss model & Two ray \\
\hline Shadowing model & Constant \\
\hline Phy layer radio type & $802.11 \mathrm{~b}$ radio \\
\hline Transmission power & $15 \mathrm{dBm}$ \\
\hline Noise factor & $7.0 \mathrm{~dB}$ \\
\hline MAC layer Link setup period & 2 seconds \\
\hline MAC layer link state period & 2 seconds \\
\hline CBR items sent & 100 \\
\hline CBR Item size & 512 bytes \\
\hline
\end{tabular}

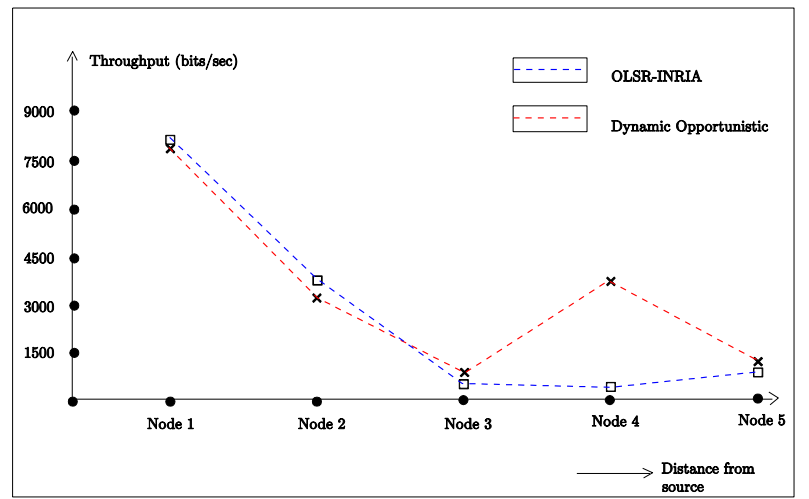

Figure 3. Throughput (bits/sec) comparison of OLSR-INRIA and Dynamic Opportunistic Routing at various nodes located at different distances.

\section{E. Performance Evaluation}

Throughput and average end-to-end delay are the most critical parameters to judge a routing protocol. Both of them were computed at various destination nodes placed at different distances in the grid. These parameters were 
then compared for two different routing protocols: Optimized Link State Routing-Institut national de recherche en informatique et en automatique (OLSR-INRIA) and our proposed Dynamic Opportunistic Routing (DOR). Referring to Fig 3, we see that

DOR gives the comparable throughput (bits/second) at nearer distance of destinations. But as we move along the X-axis (Fig. 3), at Node 4, the throughput of DOR is more than OLSR-INRIA. This was due to the comparable higher node density near Node 4 . This shows that DOR is sensitive to node density and can perform better at distant packet transmission.

Average end-to-end delay (seconds) at all destination nodes were also computed and compared, refer Fig 4. It was found that at nearer distance of transmission the delay under DOR was as more as that of OLSR-INRIA. And as we move along $\mathrm{X}$-axis, the extra throughput at Node 4 was attained at the cost of extra average end-toend delay. This shows that, if a delay is tolerable in the network, then DOR could give a better performance.

\section{Conclusion and Future Work}

In this paper, we tried to adopt dynamicity and minimize the candidate forwarder set size in multi-hop wireless routing protocol. Although we could not come up with an optimal solution, but indeed conceptually it is a new attempt. It works as good as the other proactive routing protocols at smaller distances and even better than those at longer distances. Network density plays an important role in the performance. Destinations where the density was comparably more and longer distance, our routing protocol outperformed OLSR- INRIA. The future work lies within the further optimization either by using different functionality for choosing candidate forwarder-set or using directional antennas. The extension in simulation and computing complexity in probabilistic model of network is also a future work, where we are still working on.

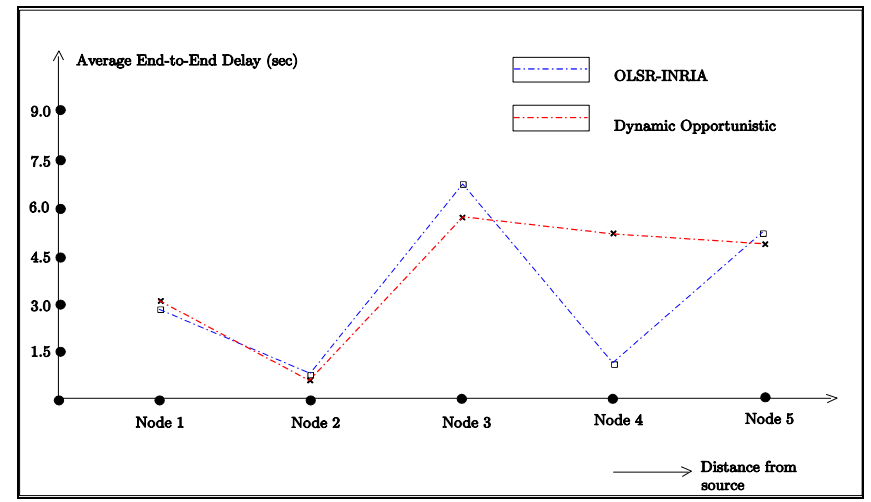

Figure 4. Average End-to-End delay (seconds) comparison of OLSR-INRIA and Dynamic Opportunistic Routing at various nodes located at different distances.

\section{References}

[1] S. Biswas and R. Morris, "ExOR: opportunistic multi-hop routing for wireless networks," Computer Communication Review, vol. 35, no. 4, p. 133, 2005.

[2] E. Rozner, J. Seshadri, Y. Mehta, and L. Qiu, "Simple opportunistic routing protocol for wireless mesh networks," in Wireless Mesh Networks, 2006. WiMesh 2006. 2nd IEEE Workshop on. IEEE, 2007, pp. 48-54.

[3] X. Mao, X. Li, W. Song, P. Xu, and K. Moaveni-Nejad, "Energy efficient opportunistic routing in wireless networks," in Proceedings of the $12^{\text {th }}$ ACM international conference on Modeling, analysis and simulation of wireless and mobile systems. ACM, 2009, pp. 253-260. 
[4] M. Naghshvar and T. Javidi, "Opportunistic routing with congestion diversity and tunable overhead," in Communications, Control and Signal Processing (ISCCSP), 2010 4th International Symposium on. IEEE, 2010, pp. 1-6.

[5] Y. Li, Y. Liu, L. Li, and P. Luo, "Local scheduling scheme for opportunistic routing," in Wireless Communications and Networking Conference, 2009. WCNC 2009. IEEE. IEEE, 2009, pp. 1-6.

[6] S. Chachulski, M. Jennings, S. Katti, and D. Katabi, "Trading structure for randomness in wireless opportunistic routing," in Proceedings of the 2007 conference on Applications, technologies, architectures, and protocols for computer communications. ACM, 2007, pp. 169-180.

[7] A. Bhorkar, B. Rao, M. Naghshvar, and T. Javidi, "An adaptive opportunistic routing scheme for wireless ad-hoc networks," in Information Theory, 2009. ISIT 2009. IEEE International Symposium on. IEEE, 2009, pp. 2838-2842.

[8] C. Luk, W. Lau, and O. Yue, "An analysis of opportunistic routing in wireless mesh network," in Communications, 2008. ICC’08. IEEE International Conference on. IEEE, 2008, pp. 2877-2883.

[9] D. Couto, D. Aguayo, J. Bicket, and R. Morris, "A high-throughput path metric for multi-hop wireless routing," Wireless Networks, vol. 11, no. 4, pp. 419-434, 2005.

[10] Q. Simulator, “Scalable Network Technologies,” Inc.[Online]. Available: www. qualnet. com.

[11] I. Alocci, S. Murphy, A. Nafaa, and J. Murphy, "Development of an IEEE 802.11 s Simulation Model for QualNet." 